\title{
Energy-Efficient and Lossless Routing Scheme for Fire Detection in Wireless Sensor Networks
}

\author{
B.Padmavathi ${ }^{1}$, N.kapileswar ${ }^{2}$, Devalla pravista sai teja ${ }^{3}$, Vijayakumar serena rajam ${ }^{4}$, \\ Dr.Polasi Phani kumar ${ }^{5}$ \\ \{padhmavb@srmist.edu.in ${ }^{1}$, kapilesn@ srmist.edu.in ${ }^{2}$, \\ ds7324@srmist.edu.in ${ }^{3}$, vr3206@srmist.edu.in ${ }^{4}$, phanikup@srmist.edu.in ${ }^{5}$ \}
}

Department of Electronics and Communication Engineering, SRM Institute of Science \& Technology, Chennai, Tamilnadu, India. ${ }^{1-5}$

\begin{abstract}
A fire detection system is one of the primary needs in forests, commercial and residential buildings. Fire detection is done using either wire-based systems or Wireless Sensor Networks (WSNs). In general, the Wireless Sensor Networks are preferred as they are faster and more accurate than the wire based fire detection systems. In Wireless Sensor Networks the fire detection can be done using various routing systems like the Wireless Fidelity (Wi-Fi), Low Power Wide Area Network (LPWAN), Wireless Metal Insulator Transition (MIT) network etc. We have adopted the Node centric lossless routing scheme which increases the load balance and minimizes the delay of relay data over the sensors to the base station using an Energy-Efficient Multilevel and Distance aware Clustering (EEMDC) for Wireless Sensor Networks. Generally, it is done using Hybrid Energy Efficient Distributive (HEED) protocol and Low-Energy Adaptive Clustering Hierarchy (LEACH) protocols. It has drawbacks with the energy levels and relaying of data. The proposed technique involves lossless and energy efficient schemes for data transmission. It uses General Self-Organized Tree-based Energy Balance (GSTEB) routing protocol, which can alter the root and renew the routing tree with a bit of delay and with consumption of less energy. Distance-Energy Cluster Structure Algorithm (DECSA) has the ability to reduce the communication complexities. Therefore, a stable and balanced load is achieved than LEACH and HEED protocols.
\end{abstract}

Keywords: Wireless Sensor Network, Fire Detection, General Self-Organized Treebased Energy Balance (GSTEB), Loss rate, Efficiency, Delay, Throughput, Efficient Low Duty Cycle (ELDC)..

\section{Introduction}

This

Fire accident is one of the most dangerous and biggest disasters. It is frequent and a powerful debacle. It obliterates properties and imperils individuals' lives. Thus, detecting a fire and putting it off is very important. This is where the fire detection system plays a major role. The traditional fire detection systems are wired based and thus have limited cable transmission distance. They also have problems in installation, construction, expansion and maintenance costs [1]. Wireless communication provides resolution to these above cited problems.

Wireless Sensor Networks (WSNs) are being used in almost all the industry these days. They are widely used in places where wired cables are ineffective and in open spaces. For 
example, monitoring a residential area or an open space for fire or smoke, medical industry, traffic control, automation, etc. They have higher mobility, flexibility and easier to install and maintain [2]. It records the environmental data with the help of small and cheap sensing nodes in it [3]. The main problem with WSN are the energy consumption and delay in relaying of data. This becomes complicated as we move on to the higher number of nodes. In WSN the energy efficiency plays a vital role as most of the sensor networks have this limitation. Increasing the performance of energy usage and extending the network life of such networks have been a long-time problem [4]. WSN with a good routing protocol will help in decreasing the consumption of node energy as its energy efficiency is limited [5]. WSN is very eminent in collecting data in collaborative method, like the embedded system and sensors are integrated foe collaborative data collection. This mainly depends on network's energy efficiency, lifespan and how balanced the network is. If the nodes have low energy the data collection and dissemination cannot be carried out. Improving the residual energy can improve the network [6].

Our proposed system overcomes all these drawbacks as it is energy efficient and has a robust routing system. This network is highly reliable and adjusting to the environment. The problem is that the sensor nodes cannot be charged again so its durability/ longevity should be high. Monitoring industrial areas require higher number of nodes as the area is usually large. The more the number of nodes higher is the accuracy of the fire detection and prevention. Surveys have proved that group networks are beneficial like the cluster networks [7]. Cluster networks play a vital role in WSNs as they make the network energy efficiency and expand the lifetime of the network. Basically, nodes are mainly two types, Low Energy Sensor Nodes (LESN) and Higher Energy Sensor Nodes (HESN). Based on the type of nodes, it will contain HESN [8]. The communication takes place by dividing the clusters into groups [9, 10]. It has three phases that are Cluster Setup Phase, Cluster Head Selection and Phase Communication Phase.

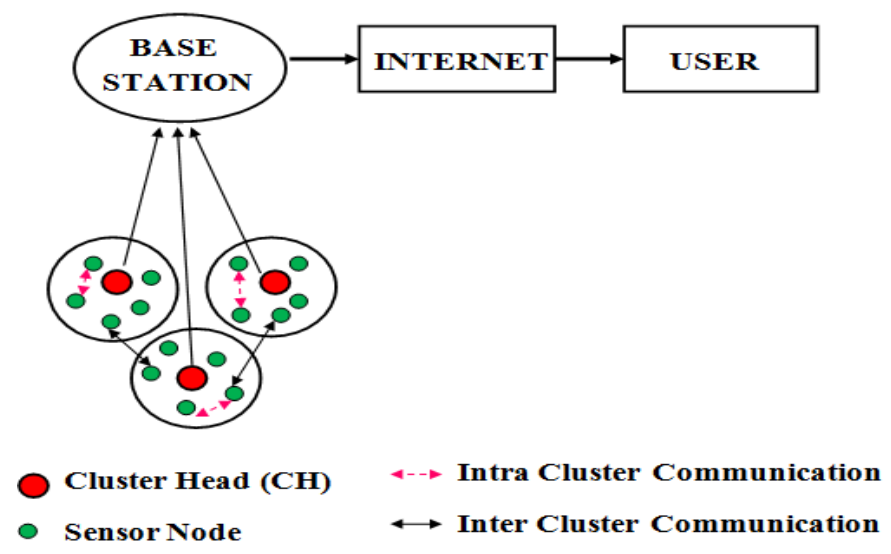

Fig. 1. Communication of cluster networks

Fig. 1 interprets the general communication of the cluster network that is the communication between the $\mathrm{CH}$, sensor node, base station, intra and inter cluster communication. Group networks have their unique ID and the middle node is selected. Each node keeps transferring the data towards the target node. The nodes at the border help communicate with the other groups. Routing protocols might differ grouped to the group. 
Communication takes place from base station to other clusters through cluster head. The selection of cluster head is done by looking at the left out residual energy [11]. In this the Energy Hole Problem arises as the cluster head is closer to the base head and it loses its energy faster due to heavy traffic there. This problem can be overcome by developing an intelligent [12] and highly efficient device that can overcome these problems and work accurately in all conditions [13].

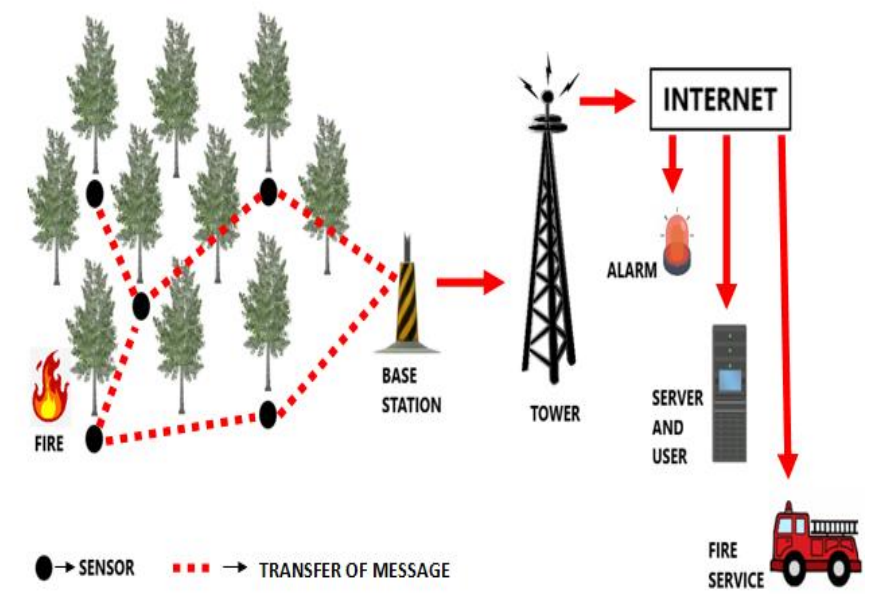

Fig. 2. Fire detection through WSNs

Fig. 2 depicts the general fire detection process through WSNs. We have proposed and implemented Energy Efficient Unequal Clustering (EEUC). This has no cluster heads and the sensor nodes of the duty cycle are changed in order to save energy. This technique works based on the pair of queues.

The rest of the paper is organized as follows. Section II provides the review of related works. Section III describes the proposed protocol and designed steps in the flow chart. Section IV inflicts the experimental results and discussions. Section V concludes the paper and references are mentioned at the end.

\section{Related Works}

The related works mentioned below depicts the Wireless Sensor Networks (WSN) and fire detection systems using different protocols which has enhanced Accuracy rate, Response time, Safety measures, Algorithms and other factors involved.

Adnan, et.al, [14] proposed a LoRa mesh network based forest fire detector. It was designed using DHT 11 and MQ2 sensor, Arduino Uno and LoRa modules. This device was able to transfer information to about $500 \mathrm{~m}$ and the RSSI received was $-136 \mathrm{dbm}$ in a closed space, but was able to transfer the data only partially in an open space. This model was able to cover about 10 hectares with only 4 LoRa modules along with 4 nodes to avoid data collisions. Dyan Quel. R, et.al, [15] developed a fire detection system for outdoor residential areas. The system was constructed by using ionization smoke sensors, GPS module [16], GSM module, Zig Bee module, ATmega328P and temperature sensor. It works within the area it has been programmed and sends alert messages to the concerned people on the detection of smoke or fire. Apart from this it also records the details of the fire accident automatically in excel file. 
Alexander [17] built a model for crown fire, which is more severe than the surface fire. The device would first detect the type of fire, that is, if its surface or crown fire, if its surface fire there is an increase in amplitude to lower frequency and if its crown fire a Gaussian trend line is formed and its frequency is from 250 to $450 \mathrm{~Hz}$. In the same way for the other types of fire a unique trend line is formed. Through this device totally 9 types of forest fires can be detected based on its unique trend line. Spectral analysis was done with 15 records and was found to be successful. This system adds on as a feather to the existing fire detection WSN system.

P.J.Y. Piera [18] developed a model for fire detection and alarm system (FDAS) to overcome the drawbacks of existing wired FDAS. It was built using a fire alarm control panel, detection and fire alarm node. They built a wireless transceiver using XBee, which makes the device flexible and has an automatic mesh network. Lab View is its control panel and it is controlled by an algorithm. A network of nodes connect and work within the programmed range and also cluster-tree automatically. LabView based GUI detects fire and takes care of the alarm. It was implemented successfully and the response time was under 10 seconds, the fastest being 0.8985 seconds and slowest being 5.018 seconds.

K. Pandey [19] suggested a WSN for early detection of fire based on Boolean-Poisson model. They worked on Poisson point process (PPP), sensor density and also the effect of wind velocity on it. The implementation and result of this model proved that the wind doesn't necessarily interrupt the fire detection, but the fire detection probability in fact increases because of wind. Shuxin Zhong [20] proposed a device for fire detection and alarm system called Wi-Fire for closed environment. This model overcomes the resultant phase and amplitude difference of Channel State Information (CSI) due to the wireless signal transmission. The CSI of RF signal was used in enhancing and improving Wi-Fire. This detects fire through radio propagation. This is carried on by data processing and extraction. This was implemented and tested, the accuracy rate was found to be $96.97 \%$.

Ferry [21] proposed a WSN based model for fire detection and home monitoring. It was built using Carbon Monoxide, temperature, humidity, air and smoke sensors. This is completely based on fire accident probability detection. If the fire accident probability goes high it automatically sends messages to the concerned person, turns on the alarm and also unlocks the house doors automatically. This was implemented, tried and tested. It was found to be quite accurate, though it had an error of only $0.3 \%$ in probability detection. Apart from this it had a problem with the algorithm and it gets restricted in open space.

E. S. Sasmita [22] proposed a model for early fire detection. It was based on Flame Sensor Module, which detects fire and it was integrated with GPS HAT which uses radio frequency to transmit data along with LoRa module. These all were further integrated with Raspberry pi. This was made as a prototype for fire detection. Information exchange for the fire detection was done through data coordinates of GPS and was sent to LoRa module. This model can also be used in disaster recovery as well. This was found to be useful, but the drawbacks being inability to send the digital location and the area of coverage.

Jimin Son [23] endeavored a Wireless Metal Insulator Transition (MIT) Fire and Alarm System to overcome the problem of high power consumption. The main components used were Critical Temperature Switch (CTS), Bluetooth low energy beacons and Microcontroller Unit (MCU). The key element being the CTS in the module. If the fire is detected CTS is shifted to low resistance and it further controls the MCU for the alarm. As the CTS activates MCU only when the fire is detected, this leads to a saving a huge amount of energy. This differentiates it from the present WSN fire detectors. Its working is completely monitored by the web pages. 
Oxsy Giandi [24] proposed a method to detect the fire and its appearance. Prediction and detection were made by the concentration of the gas leak. Data from LPG was used to detect and predict fire based on the concentration of the gas leak and the alarm would be sounded accordingly. For the fire type detection fuzzy system was used to fuse the smoke and CO data with the help of humidity and temperature sensor. The drawback of this system was MFC was not for real time use as it was slow in reading the data sent to it.

S. R. Vijayalakshmi [25] developed a system which scans the input video data and detects the fire. The main principle used was Digital Image Processing and Programming and embedded vision [26]. In the video first the frame is detected and frame conversion takes place, then the colour detection of smoke and fire takes place, then motion detection is followed by special analysis. The fake fire regions are also removed. If the fire is detected and the time interval is more than the threshold, then the alarm is activated. This method is faster to detect fire and also fully automatic in closed open space. The drawback being a bit of accuracy.

S. Wilson [27] conducted a survey on the accidents caused due to fire and its effects. They proposed a vision based fire detection system as it was found to be more accurate while the existing fire detectors, smoke detectors and wired detectors were found to be inaccurate and high false alarm rate. The vision-based fire detection system helps real-time fire detection due to the analyses of the live video footage. HSV and YCbCr have been used for fire color detection apart from this texture, shape, intensity and motion are also detected by it. This method when stimulated was found to be more effective than the existing system.

Shixiao $\mathrm{Wu}$ [28] proposed a method for forest fire detection called as a classical objective detection. They analyzed three methods of forest fire, namely R-CNN, SSD and YOLO. Out of these three methods SSD was found to be accurate and better than the other two. It had higher accuracy of fire detection and had lesser chances of false alarms. The fire was detected using 4 coordinates of SSD. Using these coordinates the frame interval is calculated and fire is detected. Muhammad. S [29] proposed a rescue system for fire accident detection. This system was designed with Arduino microcontroller, camera, smoke sensor and buzzer. In this proposed method data is collected from the camera and smoke sensors. These data are forwarded to the monitoring system which is a wireless system. This technique accurately predicts the fire and the rescue operation can be done at the earliest. Additionally, it also provides images of the accident site with the help of the image sensor.

S. Vancin [30] compared the algorithm of SEED (sleep-wake energy balanced distributed) with the existing homogeneous and heterogeneous clustering networks like LEACH, SEP, PEGASIS, CEEC, DEEC and mod-LEACH. The comparison and survey were done based on the alive nodes and their communication with the base station. This was done to check which clustering method was more accurate, energy-efficient, reliable and robust than the other clustering methods. All these methods were stimulated and compared in MATLAB. The results suggested that SEED had its peak when compared to the other methods. SEED proved to be advantageous due to its prolonged lifetime and less energy consumption.

$\mathrm{Xu}$ Jia [31] proposed a WSN based hierarchical software clustering algorithm. The cluster heads of each group along with the base heads are standardized. Metric algorithms are programmed at nodes for cluster security and accuracy. Stimulation analysis checked the rationality and accuracy. It was done with traditional algorithm and the newly designed algorithm along with the environmental parameters. The error rate, change in frequency of cluster head cluster, detection time and safety of algorithms is compared and the output graph is drawn. The error in tradition method was found to be between $40 \%-90 \%$, while that the newly designed software method was found to be $15 \%-30 \%$. Thus, this analysis proved that 
the WSN based hierarchical software clustering algorithm is more effective, accurate and can work better than the traditional algorithm. This leads to better performance of the network operation along with high safety.

S. K. M. Yendamuri [32] proposed for a three layer clustering approach rather than the existing two layers clustering approach to enhance the system and to overcome the drawbacks. For head selection at a lower level a dispersed hierarchical approach was deployed. Additionally, grid nodes were deployed for the communication with the base heads and the sensor nodes. This method was an extended version of LEACH called as EHCA. By default the $\mathrm{GH}$ were initialized. The second layer of $\mathrm{GH}$ played a vital role in transmitting messages to the first layer of $\mathrm{CH}$ and the second layer of base heads. This process reduced the total energy required, thus saving the energy. This leads to increased lifetime of the network. Therefore the stimulation proved to be successful for large networks, making them efficient and accurate along with the increased life.

M. Z. Masoud [33] proposed a Hybrid Clustering Routing Protocol (HCP) which consists of power module of LEACH and the cluster head selection protocol. It is a two phase protocol. The first phase selects the cluster heads and the second phase is responsible for clearing the traffic towards the target according to the threshold energy. In this process some end up forming clusters while some don't form clusters. This HCP uses the new proposed algorithm $\mathrm{CoN}$ for calculating the threshold value. HCP, LEACH and LEACH-T protocols were stimulated in MATLAB and their results were compared. The results proved that the proposed HCP is better than the LEACH and LEACH-T protocol as it increased the life span by $30 \%$ and reduced the energy consumption.

Jie Huang [34] proposed a multi cluster head routing system based algorithm. This method works by dividing the WSNs into clusters where the integration of data is carried out by the assistant cluster head node. It enhances the energy efficiency, which decreases the number of death nodes, hence increasing the lifespan of the network. It is a good method for realization too. For routing algorithm, it stimulates and analyzes the cluster head system. Stimulation is done based on the number of live nodes and the time of the network. The experimental analyzes and results proved that this multi cluster head algorithm was able to balance the energy with the number of nodes better. It made the network balanced, energy efficient [35] and stable.

\section{Proposed Methodology}

We proposed a protocol that is advanced to improve efficiency in broadcasting the signal to the target station. This protocol increases the network lifetime and less output delay in balancing load. The overview of the proposed protocol has been provided below. 


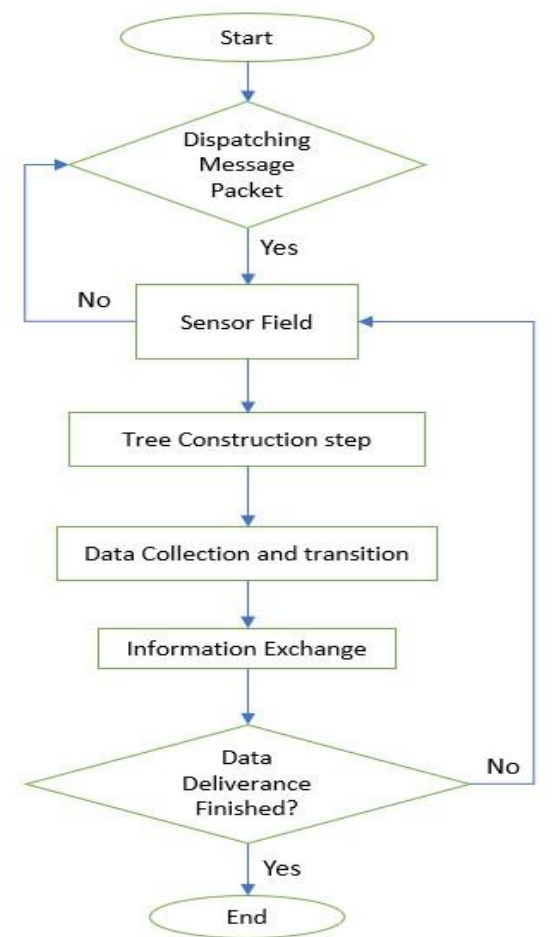

Fig. 3. Flow procedure for fire detection using GSTEB protocol

The primary goal of General Self-Organized Tree-based Energy Balance routing protocol (GSTEB) is to attain a high abundant network lifetime for different applications. It can mitigate the root and causes slight delay and modest energy utilization by remodeling the routing tree. Thus a well-balanced load is achieved distinguished with the protocols like HEED, LEACH. So, GSTEB is a self-assembled protocol that utilizes a small extent of energy.

General Self-Organized Tree-Based Energy-Balance (GSTEB) Routing Protocol is used for Wireless Sensor Networks (WSN). It can lower the whole vitality consumption and stabilize the load on WNS. In this protocol, Data Fusion takes place as individual node transmit uniform data instead of how much data gathered from its sub branches. By using this protocol, the lifetime can be increased and finally the data will be sent to the Base Station (BS).

Fig. 3 explicates that at the beginning, the cluster heads start rotating. The message packets dispatch into the sensor field. The sensor nodes accumulate the data from the base station. In the tree construction stage, the nodes start subdividing into groups and extend themselves to transfer the data. The groups comprise of message packet and collect the data from nodes, thereby information will be exchanged. Thus, the data will be passed to the required target station. If the data deliverance is not effectuated in between the medium, it turns back to the sensor field. So, it completes the entire process and reaches to destination with better throughput and packet delivery. 


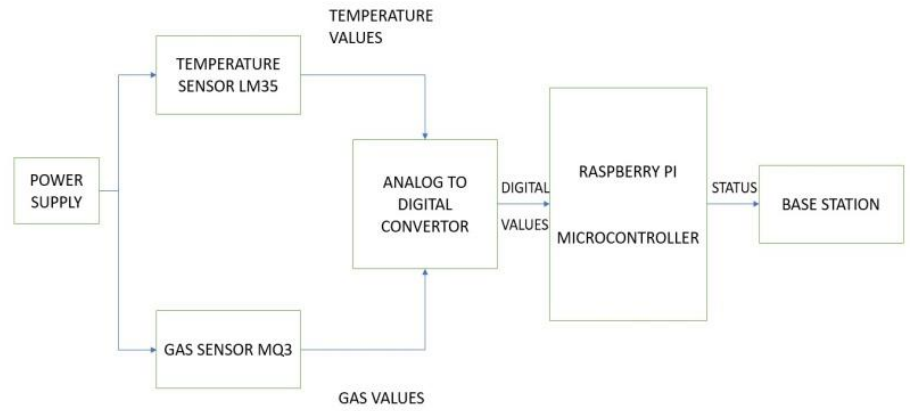

Fig. 4. Fire Detection using WSN

The above diagram Fig. 4 explains the process flow of the proposed protocol. The power supply sends the current to the temperature sensor and gas sensor. Mainly the gas sensor detects the gas and generates an emf by changing resistance. The temperature sensor identifies the temperature present in the atmosphere and converts the data into binary form. The values will be measured on analog to digital converter. The digital values are passed into the microcontroller. The Raspberry Pi Microcontroller has a clock frequency of $1.2 \mathrm{GHz}$, which improves the speed and a Processor of 64-bit quad-core ARM Cortex-A53.Hence, the base station current status will be obtained and known.

\section{Results and Discussions}

We are discussing our protocol in detail by considering the parameters Loss Ratio, Delay, Drop node, Protocol efficiency, Throughput for evaluation. Loss Ratio is usually defined in packets. When one or more packets of information fail to move from end to another end called as Packet Loss. Basically, it happens due to fallacy in transmission of data. This is majorly caused due to Interference in frequencies of signals. If two sources are placed together, one will face heavy loss of data packets. Even in Communication signals there will be lots of packet loss. This is caused by Bit Error Rate (BER).

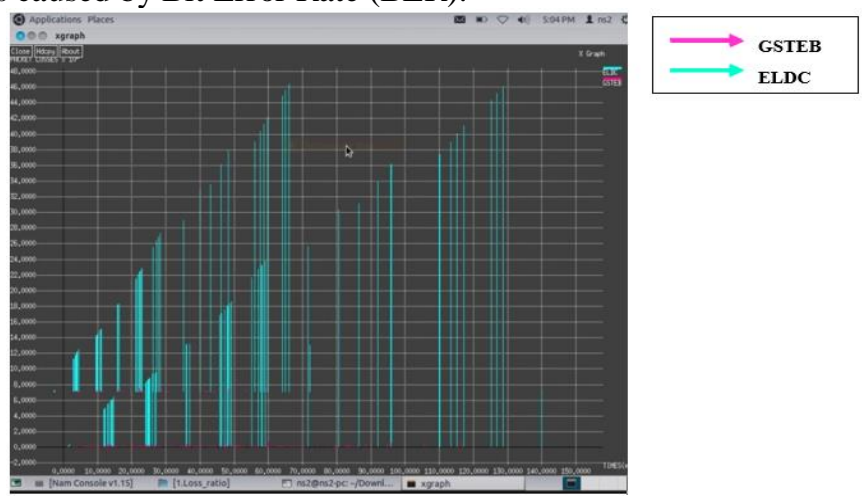

Fig. 5. Loss Ratio Output

The above Fig. 5 shows the relationship between and time and Loss and the difference between loss parameter between the EDLC and GSTEB protocol where the red line indicates 
the GSTEB and green line indicates ELDC. It is evident that loss is reduced by using GSTEB protocol.

Delay is the time taken to operate certain packets of data. In depth we can say that the packet travelling from one node to another node and finally gets reset at destination is known as delay (1). There are many types of delays involved in operating the data packets. Loss of data packets depends on the buffer and finally it affects the network performance. Mainly transmission delay plays a major role in transmitting packets to the medium.

$$
d_{\text {Nodal }}=d_{\text {Proc }}+d_{\text {Queue }}+d_{\text {Tran }}+d_{\text {Prop }}
$$

Fig. 6 shows the relationship between and time and delay and difference in the delay parameter between the EDLC and GSTEB protocol where the red line indicates the GSTEB and green line indicates ELDC. It is evident that the delay is reduced by using GSTEB protocol.

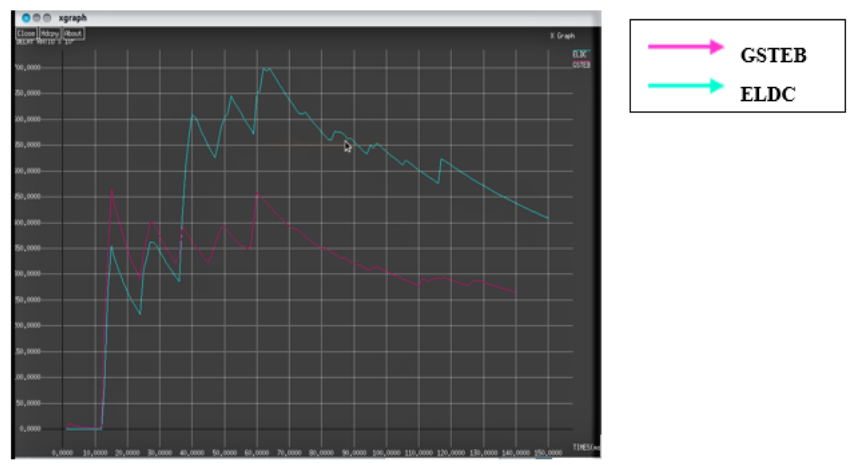

Fig. 6. Delay Output

The corresponding location within the sensors and sink at the end to end delay will be vigorous accompanying with cluster heads 'resource allocations. The data packets are transferred to an extreme delay area unit born in the real-time traffic. The timeline sharing of every $\mathrm{CH}$ for native and inter-cluster traffic transmissions are provided. They are associated with nursing analytical model, which are created from entirely different clusters and manifested to search out the dissemination of the end-to-end transmission delay for packets. Thus the packet drop rate is plagiarized from the outcome.

Later, a tentative theme is mapped out to put together notice the timeline allocations of all the CHs during a WSN to realize the minimum and the balanced packet drop rate for traffic originated. Simulation result area unit given to examine and prove the analysis and potency of the designed $\mathrm{CH}$ timeline share them. 


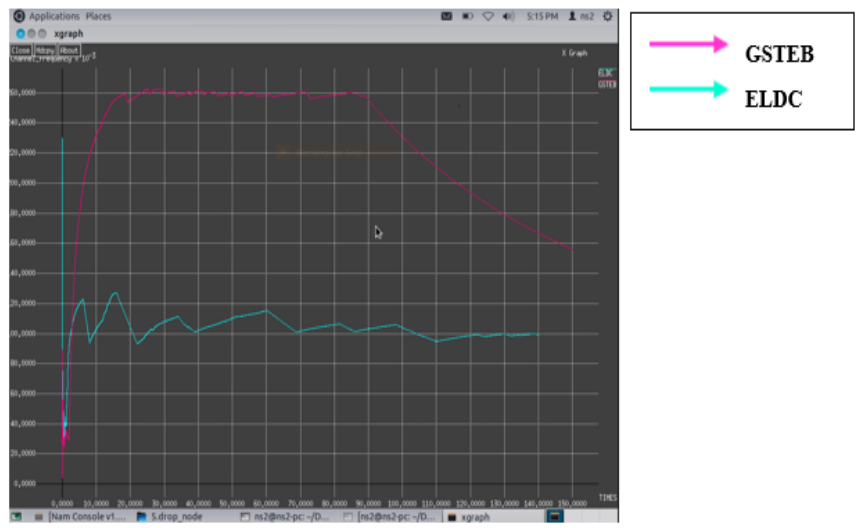

Fig. 7. Drop Node Output

Fig. 7 shows the relationship between and time and drop node parameter and the difference between drop node parameter between the EDLC and GSTEB protocol where the red line indicates the GSTEB and green line indicates ELDC. It is evident that drop node parameter is reduced by using GSTEB protocol.

Efficiency (2) was examined for different packet sizes as well as different transaction setups. Analysis was performed on a transaction base and Substantial humiliation in protocol efficiency. The efficiency calculation based on the below parameters.

$$
E_{f f}=\frac{N D}{\operatorname{Tr} D * N D R}
$$

ND $=$ Amount of Network Data*60, 400- and 1500-bytes Data Packets

$\operatorname{TrD}=$ Transaction duration

*DFC (Data-Ack and RTS-CTS-Data-Ack)

*PCF (CF-Poll $\{+$ Data $\}-\mathrm{CF}-$ Ack $\{+$ Data $\})$

NDR $=$ Nominal Data Rate

*2Mbps, $11 \mathrm{Mbps}$, 24Mbps and 54Mbps

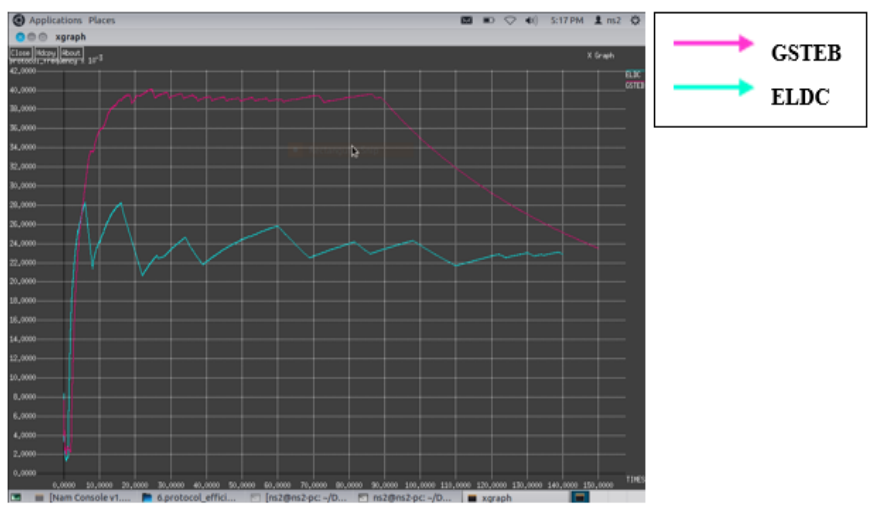

Fig. 8. Protocol Efficiency Output

The above Fig. 8 shows the relationship between and time and protocol efficiency and the difference between protocol efficiency between the EDLC and GSTEB protocol where the red line indicates the GSTEB and green line indicates ELDC. The designed protocol achieves 
98.90\% efficiency, utilization respectively, over the ELDC. It is evident that protocol efficiency is enhanced by using GSTEB protocol.

Throughput is the rate of the message that is successfully delivered over a communication medium. It is expressed in Bits per Second (bps). It is the data transmitted over a unit of time and data passing through a medium. The estimated maximum throughput will be shorter at the layer above it, because every layer appends a protocol for assigned maximum throughput at a lower layer.

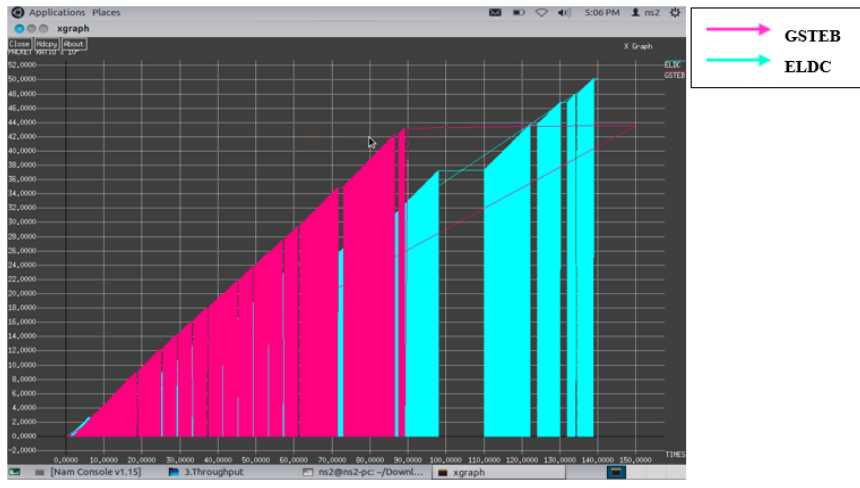

Fig. 9. Throughput Output

Fig. 9 shows the relationship between and time and throughput and difference between throughput between the EDLC and GSTEB protocol where the red line indicates the GSTEB and green line indicates ELDC. The throughput achieved for the GSTEB Protocol is $355.56 \mathrm{Kbps}$ over ELDC. It is evident that throughput is enhanced by using GSTEB protocol.

\section{Conclusion}

Thus, the system with DECSA protocol along with GSTEB protocol for fire detection based on WSN to overcome the delay of relay data, decreases energy consumption and increases the stability of the system. DECSA decreases the interfacing difficulties and GSTEB protocol enhances the network lifetime and makes the load more balanced. Also, the increased number of nodes enhances the efficiency.

We simulated the GSTEB and ELDC protocol in the software and analyzed the results of the proposed system which proved that the loss ratio, delay and drop node was lower in GSTEB protocol when compared to the ELDC protocol. Also, the GSTEB protocol achieved 98.90\% efficiency and 355.56 Kbps throughput over the ELDC protocol. Thus the efficiency and throughput are enhanced in GSTEB protocol. Therefore, the results show that the proposed algorithm and protocol overcomes the drawbacks and proves to be more stable and balanced than LEACH and HEED protocols. However, the results have been stimulated and studied only for the static environment. The dynamic environment will be considered and worked on in the future.

\section{References}

[1] L. Huang, H. Chang, C. Chen, and C. Kuo, "A ZigBee-based monitoring and protection system for building electrical safety,” Elsevier Energy and Buildings, Vol.43, no.6, pp. 1418-1426, Jun. 2011. 
[2] K. Nellore, and G.P. Hancke, "A survey on urban traffic management system using wireless sensor networks," Sensors, Vol. 16, no. 2, pp. 1-25, Jan. 2016.

[3] L. Peng, et.al. "Research on data privacy protection algorithm with homomorphism mechanism based on redundant slice technology in wireless sensor networks", China Communications, Vol. 16, no.5, pp. 158-170, Jun 2019

[4] J Hao, G. Guan, B. Zhang, and C. Li, "An Energy-Efficient On-Demand Multicast Routing Protocol for Wireless Ad Hoc and Sensor Networks", IEEE International conference on Global Communications, pp. 4650-4655, Dec. 2013.

[5] C. Jiang, Y. Ren, Y. Zhou, and H. Zhang, "Low-energy Consumption Uneven Clustering Routing Protocol for Wireless sensor Networks," 8th IEEE International Conference on Intelligent HumanMachine Systems and Cybernetics, pp.187-190, Aug. 2016.

[6] A. Aalavandhar, and A. Arjunan, "A Comparative Study of Different Cluster Mechanism for Wireless Sensor Networks," Proceedings of the Third IEEE International Conference on Electronics Communication and Aerospace Technology, pp. 781-785, Apr. 2019

[7] M. Garcia, S. Sendra, J. Lloret, and A. Canovas, "Saving energy and improving communications using cooperative group-based wireless sensor networks," Telecommunication Systems," Vol.52, no.4, pp. 2489-2502, Apr.2013.

[8] S. Misra, and R. Kumar, "A Literature Survey on Various Clustering Approaches in Wireless Sensor Network," IEEE 2nd International Conference on Communication, Control and Intelligent Systems, pp.18-22, Mar. 2017.

[9] S. Soniiand, and B. Dey, "Dynamic Selection of Cluster Head in Cluster of Cluster Heads within the Cluster in Heterogeneous Wireless Sensor Network", IEEE International Conference on Advanced Communication Control and Computing Technologies (ICACCCT), pp. 877-881, Jan. 2015.

[10] S.K. Gottapu, et.al. "Maximizing cognitive radio networks throughput using limited historical behavior of primary users," IEEE Access, Vol. 6, pp. 12252-12259, Mar. 2018.

[11] M. Garcia, S. Sendra, J. Lloret, and A. Canovas, "Saving energy with cooperative group-based wireless sensor networks, International Conference on Cooperative Design", Cooperative Design, Visualization, and Engineering, pp. 73-76, 2010.

[12] K. Nellore, and G.P. Hancke, "Traffic management for emergency vehicle priority based on visual sensing," Sensors, Vol. 16, no. 11, pp. 1-22, Nov. 2016.

[13] E. Klaoudatou, E. Konstantinou, G. Kambourakis, and S. Gritzalis, "A survey on cluster-based group key agreement protocols for WSNs," IEEE Communications Surveys \& Tutorials, Vol.13, no.3, pp. 429-44, July. 2010

[14] A.E.U. Salam, A. Arifin, and M. Rizhal, "Forest Fire Detection using LoRa Wireless Mesh Topology", The 2nd East Indonesia Conference on Computer and Information Technology, pp. 184-187, Oct. 2019.

[15] D.Q.R Elizalde, R.J.P. Garcia, M.M.S. Mitra, and R.G. Maramba, "Wireless Automated Fire Detection System on Utility Posts Using ATmega328P," IEEE 10th International Conference on Humanoid, Nanotechnology, Information Technology, Communication and Control, Environment and Management (HNICEM), pp. 1-5, Mar. 2019.

[16] P. Shivam, et.al, "Socio-medic Drone with Integrated Defibrillator," In Micro-Electronics and Telecommunication Engineering, pp. 659-668. Springer, Singapore, 2020.

[17] A.A. Khamukhin, and S. Bertoldo, "Spectral Analysis of Forest Fire Noise for Early Detection using Wireless Sensor Networks", International Siberian Conference on Control and Communications (SIBCON), pp. 1-4, Jun.2016

[18] P.J.Y. Piera and J.K.G. Salva, "A Wireless Sensor Network for Fire Detection and Alarm System," 7th International Conference on Information and Communication Technology (ICoICT), pp. 1-5, Sep.2019.

[19] K. Pandey, and A. Gupta, "Modelling and Analysis of Wildfire Detection using Wireless Sensor Network with Poisson Deployment," IEEE International Conference on Advanced Networks And Telecommunication Systems (ANTS), pp. 1-6, 2018. 
[20] S. Zhong, Y. Huang, R. Ruby, L. Wang, Y. Qiu, and K. Wu, "Wi-Fire: Device-free Fire Detection using WiFi Networks," IEEE International Conference on Communications (ICC), pp. 1-6, Jul.2017

[21] F. A. Saputra, et.al. "Prototype of Early Fire Detection System for Home Monitoring Based on Wireless Sensor Network," International Electronics Symposium on Engineering Technology and Applications (IES-ETA), pp. 39-44, Dec. 2017.

[22] E.S. Sasmita, M. Rosmiati, and M.F. Rizal, "Integrating Forest Fire Detection with Wireless Sensor Network Based on Long Range Radio," International Conference on Control, Electronics, Renewable Energy and Communications (ICCEREC), pp. 222-225, May 2019.

[23] J. Son, et.al. "Ultralow Power Wireless-Fire-Alarm-System using a VO2-Based Metal-InsulatorTransition Device," 16th Annual IEEE International Conference on Sensing, Communication, and Networking (SECON), pp. 1-2, Sep.2019

[24] O. Giandi, and R. Sarno, "Prototype of fire symptom detection", International Conference on Information and Communications Technology, pp. 489-494, Mar 2018.

[25] S.R. Vijayalakshmi, and S. Muruganand, "Fire alarm based on spatial temporal analysis of fire in video," Proceedings of the Second International Conference on Inventive Systems and Control (ICISC 2018), pp. 104-109, 28 Jun.2018.

[26] B. Akhıl, et al. "A Smartphone Controlled Fertilizing and Plant Watering Garduino," International Journal of Applied Mathematics Electronics and Computers, Vol. 8, no. 4, pp. 173-178, Dec 2020.

[27] S. Wilson, et.al. "A Comprehensive Study on Fire Detection", Proc. IEEE Conference on Emerging Devices and Smart Systems (ICEDSS 2018), pp. 242-246, Nov. 2018.

[28] S. Wu, and L .Zhang, "Using Popular Object Detection Methods for Real Time Forest Fire Detection," 11th International Symposium on Computational Intelligence and Design, pp. 280-284, 25 Apr. 2019.

[29] M.S.A. Azmil, et.al. "Wireless Fire Detection Monitoring System for Fire and Rescue Application," IEEE 11th International Colloquium on Signal Processing \& its Applications (CSPA), pp. 84-89, 27 Aug. 2015.

[30] S. Vancin, and E. Erdem, "Performance Analysis of the Energy Efficient Clustering Models in Wireless Sensor Networks," 24th IEEE International Conference on Electronics, Circuits and Systems (ICECS), pp. 247-251, 15 Feb. 2018.

[31] X.Jia, and G.Yang, "Research on Hierarchical Software Clustering Algorithm Based on Wireless Network", International Conference on Computer Network, Electronic and Automation (ICCNEA), pp. 284-290, 28 Nov.2019.

[32] S.K.M. Yendamuri, et.al. "An Improved Three-Layer Clustering Hierarchy for Wireless Sensor Networks: A Proposed Framework," 9th International Conference on Computing, Communication and Networking Technologies (ICCCNT), pp. 1-6, Jul. 2018.

[33] M.Z. Masoud, et.al. "To Cluster or Not to Cluster: A Hybrid Clustering Protocol for WSN," IEEE Jordan International Joint Conference on Electrical Engineering and Information Technology (JEEIT), pp. 678-682, May. 2019.

[34] J. Huang, "Research on Balanced Energy Consumption of Wireless Sensor Network Nodes Based on Clustering Algorithm", International Conference on Computer Network, Electronic and Automation, pp. 300-304, Dec.2017.

[35] K. Nellore, et.al, "A fast information dissemination system for emergency services over vehicular ad hoc networks," IEEE International Conference on Energy, Communication, Data Analytics and Soft Computing (ICECDS), pp. 236-241, August 2017. 